\title{
Vibration Transfer Matrix Model of Multilayered Wave-attenuation Media of Warship
}

\author{
Wei $\mathrm{Ai}^{*}$ \\ Dept. of Navigation, Dalian Naval Academy \\ Key Laboratory of Military navigation \\ Liaoning, Dalian, 116018, China \\ E-mail: awtg2009@126.com \\ * Corresponding Author \\ Wei Chi \\ Dept. of Navigation, Dalian Naval Academy \\ Key Laboratory of Military navigation \\ Liaoning, Dalian, 116018, China
}

\author{
Liang'an Jin, \\ Dept. of Navigation, Dalian Naval Academy, \\ Key Laboratory of Military navigation \\ Liaoning, Dalian, 116018, China \\ e-mail: jinliangan@163.com
}

\begin{abstract}
Warship vibration isolation technology of multilayered wave-attenuation is based on new mechanism. In view of the influence of vibration transfer efficiency, which belongs to statics contains, this paper presents a two-dimensional transfer matrix model in the condition of oblique incidence, and gets derivation formulas of transmission and reflection coefficient. The construction of the transfer matrix model is based on the representation analysis of structure vibration energy and acoustic boundary conditions, and the new model is to study the implementation effect on vibration isolation technology of multilayered wave-attenuation. Then, by designing specialized experimental equipment and analyzing the results, the excellent implementation effect on multilayered wave-attenuation vibration isolation is verified. In addition, the calculation accuracy of the new model has been verified by contrastively analyzing the results of the new model with other literature.
\end{abstract}

Keywords-Multilayered Media; Transfer Matrix; Vibration Energy; Reflection Coefficient; Transmission Coefficient

\section{INTRODUCTION}

With continuous improvement of the status of marine rights and interests in national strategy, warship is to develop in the direction of large-scale, high-speed, integration, and concealment. Now underwater acoustic equipment operating distance is more and more far, the application of acoustic homing and fuzing weapons arebecoming more and more widely, putting forward higher requirements for ship acoustic stealth technology. Therefore, navies bring the warship acoustic stealth technology into the focus of the research in high speed of arms race. At intermediate or low ship speed, main structural vibrations are originated from mechanical equipment, shafting system and weapon launcher motion, which are transmitted through supporting foundation, piping system and air to the hull, and then, the excited hull vibrations radiate wave energy into
water[1].Research shows that, main energy of structural vibrations transmit through supporting foundation, and the most effective method to control it is to implement vibration isolation technology[2].

Vibration isolation technology has excellent characteristics of high damping capacity and convenient installation, which makes scholars focus the research on core actuators and vibration isolator development, and a lot of achievements have been obtained [3-4]. He et al [5] independently developed pneumatic vibration isolators for marine main engine, which had successfully realized the engineering application, and among them, type JYQN had excellent performance and applicability. Zhang et al [5] filled pneumatic vibration isolator with elastic and liquid to overcome problems of air leakage and instability, which lifted the restrictions of position monitoring and maintaining equipment. Because of filling vibration isolator with solid-liquid mixed media possessing obvious advantages in low-frequency and heavy-duty vibration isolation, a lot of literatures carry out researches on this field[7-9], and it is obvious that vibration isolation technology making full use of mixed media will be applied widely.

Literatures [10-11] put forward a new method to improve warship vibration isolation efficiency by filling closed isolator with multilayered wave-attenuation mixed media, thus, the vibration wave energy can be attenuated to the maximum extent. Comparing with filling single medium, the distinctive features of the new method act multiple wave-attenuation mechanisms comprehensively, including reflection, transmission, absorption, exciting liquid surface wave, etc. Therefore, researchers can say that the new method is able to achieve the purpose of high efficiency vibration isolation on root. Therefore, in order to provide important measure index and theoretical basis of wave-attenuation efficiency for improving vibration isolation theory, 
Researchers present a two-dimensional transfer matrix and get derivation formulas of transmission and reflection coefficient.

\section{TRANSFER MATRIX MODEL DERIVATION}

\section{A. Acoustic energy relationship and characterization}

Medium volume element in sound field is $\Delta V$, sound pressure is $p_{0}$, density is $\rho_{0}$, volume element kinetic energy and potential Energy are respectively $E_{k}=\frac{1}{2} \Delta V p_{0} v^{2}$ and $E_{p}=\frac{\Delta V}{2 \rho_{0} c_{0}^{2}} p^{2}$, where $v$ is particle vibration velocity, $c_{0}$ is sound velocity in medium. Therefore, sound energy of volume element can be shown as,

$$
E=E_{k}+E_{p}=\frac{\Delta V \rho_{0}}{2}\left(v^{2}+\frac{p^{2}}{\rho_{0}^{2} c_{0}^{2}}\right)
$$

Sound energy density equation is,

$$
\varepsilon=\frac{E}{\Delta V}=\frac{\rho_{0}}{2}\left(v^{2}+\frac{p^{2}}{\rho_{0}^{2} c_{0}^{2}}\right)
$$

Let sound pressure

be $p(t, x)=p_{a} e^{j(w t-k x)}$, particle vibration velocity be $v(t, x)=v_{a} e^{j(w t-k x)}$, among expressions, $p_{a}$ and $v_{a}$ are amplitudes of pressure expression and particle vibration velocity. Then, real parts of $p(t, x)$ and $v(t, x)$ are substituted into Formula 1, and instantaneous value of sound energy in unit volume is described as Formula 3,

$$
\begin{aligned}
E & =\frac{\Delta V \rho_{0}}{2}\left[\frac{p_{a}^{2}}{\rho_{0}^{2} c_{0}^{2}} \cos ^{2}(w t-k x)+\frac{p_{a}^{2}}{\rho_{0}^{2} c_{0}^{2}} \cos ^{2}(w t-k x)\right] \\
& =\frac{\Delta V p_{a}^{2}}{\rho_{0} c_{0}^{2}} \cos ^{2}(w t-k x)
\end{aligned}
$$

Average values of sound energy and sound energy

$$
\text { density during period } T \text { are } \bar{E}=\frac{1}{T} \int_{0}^{T} E d t=\frac{\Delta V p_{a}^{2}}{2 \rho_{0} c_{0}^{2}} \text {, }
$$$$
\bar{\varepsilon}=\frac{p_{a}^{2}}{2 \rho_{0} c_{0}^{2}}
$$

According to the definition of sound intensity, which is average acoustic energy flow of unit area in vertical direction, the relationship among sound intensity of sound energy, average value of sound energy and average value of sound energy density are shown as Formula 4,

$$
I=\frac{c_{0}}{\Delta V T} \int_{0}^{T} E d t=\frac{c_{0}}{\Delta V} \bar{E}=c_{0} \bar{\varepsilon}=\frac{p_{a}^{2}}{2 \rho_{0} c_{0}}=\frac{\rho_{0} c_{0} v_{a}^{2}}{2}
$$

In the application of practical engineering, total vibration energy at steady state and average value of sound energy density are generally used to evaluate structures vibration, but both of them cannot be measured directly. Thus, vibration acceleration is chosen to be the measuring parameter in vibration level assessment. In the interval of $\left(t_{1}, t_{2}\right)$, mean square value of energy signal is $A=\int_{t_{1}}^{t_{2}} a^{2}(t) d t$, and the damping effect of the vibration isolation measures can be described as Formula 5, which is defined as VLDA (vibration level difference of acceleration).

$$
T=10 \lg \frac{\frac{1}{n} \sum_{i=1}^{n} A_{i}^{2}}{\frac{1}{n} \sum_{i=1}^{n} A_{0 i}^{2}}=10 \lg \frac{\bar{A}^{2}}{\bar{A}_{0}^{2}}
$$

Among it, $\bar{A}^{2}$ is mean square value of post-isolation vibration acceleration, $\bar{A}_{0}^{2}$ is mean square value of pre-isolation vibration acceleration. When single degree of freedoms structure is in forced vibration, the expression of vibration energy at steady state can be described as $W=\frac{m \bar{A}^{2}}{\omega^{2}}$, among it, $m$ is the mass of single degree of freedoms structure, and $\omega_{\text {is circular }}$ frequency of external force. Therefore, by modeling a two-dimensional transfer matrix in the condition of oblique incidence and getting transmission coefficient, the energy exchange relations of multilayered wave-attenuation mixed media can be obtained. It is obvious that sound intensity transmission coefficient is an important index to measure wave-attenuation efficiency.

\section{B. Modeling transfer matrix of multilayered media}

Longitudinal wave can be generated in multilayered fluid system with plane wave oblique incidence, and acoustic boundary conditions, which continuities of sound pressure and normal mode velocity, are satisfied based on analysis of Fig .1. Therefore, the sound pressure field of layer ${ }^{i}$ and layer $i+1$ can be described as Formula 6 and Formula 7, among it, $i$ represents the layer number, and it is to use $D_{i-1}$ as coordinate axis $x$ of layer $^{i}, \quad P^{i}=P_{i}^{i}+P_{r}^{i}, P^{i+1}=P_{i}^{i+1}+P_{r}^{i+1}$,

$$
\begin{aligned}
& \left\{\begin{array}{l}
P_{i}^{i}=p_{t a}^{i} e^{\left.j\left[w t-k_{i}\left(x-D_{i-1}\right) \cos \theta_{i}^{i}-k_{i} y \sin \theta_{i}^{i}\right)\right]} \\
P_{r}^{i}=p_{r a}^{i} e^{\left.j\left[w t+k_{i}\left(x-D_{i-1}\right) \cos \theta_{r}^{i}-k_{i} y \sin \theta_{r}^{i}\right)\right]}
\end{array}\right. \\
& \left\{\begin{array}{l}
P_{i}^{i+1}=p_{t a}^{i+1} e^{\left.j\left[w t-k_{(i+1)}\left(x-D_{i}\right) \cos \theta_{t}^{i+1}-k_{(i+1)} y \sin \theta_{t}^{i+1}\right)\right]} \\
P_{r}^{i+1}=p_{r a}^{i+1} e^{\left.j\left[w t+k_{(i+1)}\left(x-D_{i}\right) \cos \theta_{t}^{i+1}-k_{(i+1)} y \sin \theta_{t}^{i+1}\right)\right]}
\end{array}\right.
\end{aligned}
$$

According to Formula 6, Formula 7 and motion equations as Formula 8 , the velocity potential can be obtained which are described as Formula 9 and Formula 10 , 


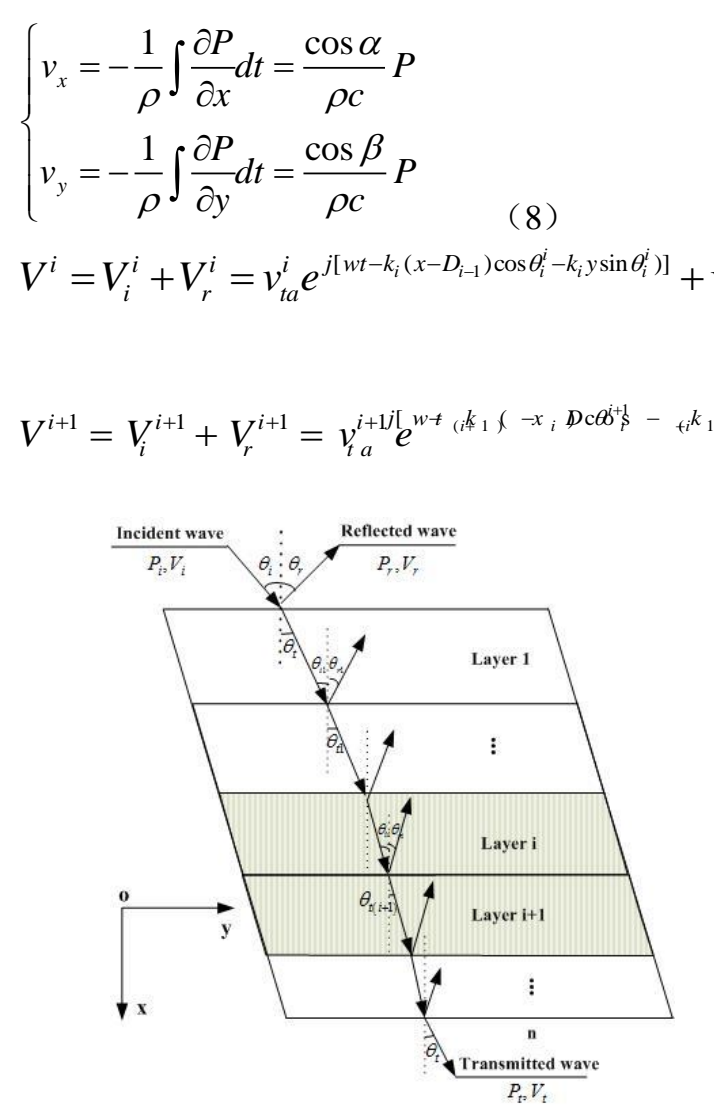

Figure 1. Sketch map of multilayered system subjected to oblique incidence

On the simultaneous of Formula 6 and Formula 7 , Formula 9 and Formula 10, important equations can be obtained, which are $A p_{t a}^{i}+B p_{r a}^{i}=p_{t a}^{i+1}+p_{r a}^{i+1}$ and $A v_{t a}^{i}+B v_{r a}^{i}=v_{t a}^{i+1}+v_{r a}^{i+1} \quad, \quad$ among them, $A=e^{-j k_{i} d_{i} \cos \theta_{i}^{i}}, B=e^{j k_{i} d_{i} \cos \theta_{i}^{i}}, d_{i}=D_{i}-D_{i-1}$. The relations of sound pressure and particle vibration velocity in layer $i$ and layer $i+1$ is as follows,

$$
\begin{aligned}
& \left\{\begin{array}{l}
p_{t a}^{i+1}=A\left(\frac{Z_{i+1}}{Z_{i}}+1\right) p_{t a}^{i}+B\left(1-\frac{Z_{i+1}}{Z_{i}}\right) p_{r a}^{i} \\
p_{r a}^{i+1}=A\left(1-\frac{Z_{i+1}}{Z_{i}}\right) p_{t a}^{i}+B\left(\frac{Z_{i+1}}{Z_{i}}+1\right) p_{r a}^{i}
\end{array}\right. \\
& \left\{\begin{array}{l}
v_{t a}^{i+1}=A\left(\frac{Z_{i}}{Z_{i+1}}+1\right) v_{t a}^{i}+B\left(\frac{Z_{i}}{Z_{i+1}}-1\right) v_{r a}^{i} \\
v_{r a}^{i+1}=A\left(\frac{Z_{i}}{Z_{i+1}}-1\right) v_{t a}^{i}+B\left(\frac{Z_{i}}{Z_{i+1}}+1\right) v_{r a}^{i}
\end{array}\right.
\end{aligned}
$$

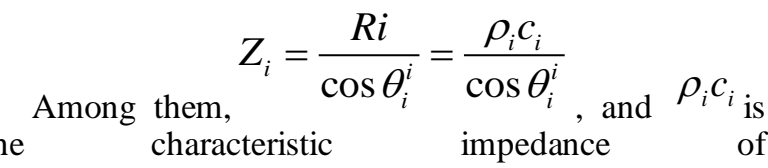
layer $i \quad \theta_{i}^{i}=\theta_{r}^{i}, \quad \theta_{t}^{i+1}=\theta_{r}^{i+1}$, $\sin \theta_{i}^{i} / \sin \theta_{t}^{i+1}=k_{i+1} / k_{i}=c_{i} / c_{i+1}, k_{i}$ is the wave number of layer ${ }^{i}$. Modifying Formula 11 and Formula
12 into matrix form $\left[\begin{array}{c}p_{t a}^{i+1} \\ v_{t a}^{i+1}\end{array}\right]=\frac{1}{2} \mathbf{H}\left[\begin{array}{c}p_{t a}^{i} \\ v_{t a}^{i}\end{array}\right]$ $\left[\begin{array}{c}p_{r a}^{i+1} \\ v_{r a}^{i+1}\end{array}\right]=\frac{1}{2} \mathbf{J}\left[\begin{array}{c}p_{r a}^{i} \\ v_{r a}^{i}\end{array}\right]$

$\mathbf{H}$ and $\mathbf{J}$ are transfer matrix of transmission and reflection coefficient in layer $i$ and layer $i+1$, to expand $\mathbf{H}$ and $\mathbf{J}$ as follows:

$$
\begin{aligned}
& \mathbf{H}=\left[\begin{array}{ll}
\left(\frac{Z_{i+1}}{Z_{i}}+1\right) e^{-j k_{i} d_{i} \cos \theta_{i}^{i}} & \left(1-\frac{Z_{i+1}}{Z_{i}}\right) e^{j k_{i} d_{i} \cos \theta_{i}^{i}} \\
\left(\frac{Z_{i}}{Z_{i+1}}+1\right) e^{-j k_{i} d_{i} \cos \theta_{i}^{i}} & \left(\frac{Z_{i}}{Z_{i+1}}-1\right) e^{j k_{i} d_{i} \cos \theta_{i}^{i}}
\end{array}\right] \\
& \mathbf{J}=\left[\begin{array}{ll}
\left(1-\frac{Z_{i+1}}{Z_{i}}\right) e^{-j k_{i} d_{i} \cos \theta_{i}^{i}} & \left(1+\frac{Z_{i+1}}{Z_{i}}\right) e^{j k_{i} d_{i} \cos \theta_{i}^{i}} \\
\left(\frac{Z_{i}}{Z_{i+1}}-1\right) e^{-j k_{i} d_{i} \cos \theta_{i}^{i}} & \left(\frac{Z_{i}}{Z_{i+1}}+1\right) e^{j k_{i} d_{i} \cos \theta_{i}^{i}}
\end{array}\right]
\end{aligned}
$$

\section{SOUND INTENSITY TRANSFER COEFFICIENT}

In the multilayered system subjected to normal incidence plane waves, which is $\theta_{i}=0$, transfer matrix results of Formula 13 and Formula 14 in layer $i$ and layer $i+1$ have the same form with sound pressure transfer matrix ${ }^{A_{i}}$ of literature [12]. Then, rewriting

Formula 13 into a general form as

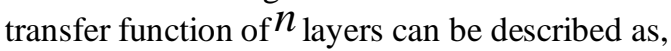

$$
p_{r a}^{n}=\left(\frac{1}{2}\right)^{n} H_{21}^{1} \cdot H_{21}^{2} \cdots H_{21}^{n} p_{t a}^{0}+\left(\frac{1}{2}\right)^{n} H_{22}^{1} \cdot H_{22}^{2} \cdots H_{22}^{n} p_{r a}^{0}
$$

Then, substituting the known conditions ${ }_{\text {as }} p_{r a}^{0}=P_{r}, p_{t a}^{0}=P_{i}, \quad p_{r a}^{n}=0$ into Formula 14, sound pressure reflection coefficient $\xi$ and transmission coefficient ${ }^{\eta}$ can be resolve to, 


$$
\begin{gathered}
\xi=\frac{\left|P_{r}\right|^{2} / 2 R_{1}}{\left|P_{i}\right|^{2} / 2 R_{1}}=\left|\frac{\prod_{l=1}^{n} H_{21}^{l}}{\prod_{l=1}^{n} H_{22}^{l}}\right|^{2} \\
\eta=\frac{\left|P_{t}\right|^{2} / 2 R_{n}}{\left|P_{i}\right|^{2} / 2 R_{1}}=\left(\frac{1}{2}\right)^{2 n} \frac{R_{1}}{R_{n}}\left|\prod_{l=1}^{n} H_{11}^{l}-\frac{\prod_{l=1}^{n}\left(H_{12}^{l} \cdot H_{21}^{l}\right)}{\prod_{l=1}^{n} H_{22}^{l}}\right|^{2}
\end{gathered}
$$

In the process of sound waves transfer in fluid, the medium features of viscosity, heat conduction and relaxation effect show significant energy attenuation, thus, it is needed to turn the expressions of sound velocity and wave number which are closely related to characteristic impedance into form of complex. Complex sound velocity is $\tilde{c}=c\left(1-i \varepsilon_{c}\right)$ and complex wave number is $\tilde{k}=k\left(1+i \varepsilon_{k}\right)$, among them, $\varepsilon_{c}$ and $\varepsilon_{k}$ are the loss factors.

\section{EXPERIMENTAL RESULTS AND MODEL TEST}

According to derivations and actual measuring demand, the test system of vibration isolation technology of multilayered wave-attenuation is designed, the schematic diagram of which is shown in Fig .2. The exciter is precision speed regulating motor of $\mathrm{LN} 30 / 2$ with vibration frequency of $0-300 \mathrm{~Hz}$, and the closed isolator is filled with multilayered wave-attenuation media of water and dimethicone. Upper and lower mass both are $5 \mathrm{Kg}$ rigid structures, vibration sensor adopts three-axis acceleration chip with adjustable measuring range, and the testing equipment is the STC12C5A60S2 series MCU. Fig .3 shows the VLDA of filling isolator with different media. From Fig .3, it can be seen that the VLDA increases firstly and then decreases with the increase of water filling amount, when water mass reaches $0.4 \mathrm{~kg}$, the max VLDA is $9.61 \mathrm{~dB}$. The VLDA variation filling with dimethicone is consistent with that of filling with water, and the max VLDA is $10.2 \mathrm{~dB}$. When filling with mixed media of water and dimethicone, the max VLDA reaches $14.08 \mathrm{~dB}$ at $0.5 \mathrm{~kg}$ total mass, thus, filling with multilayered wave-attenuation media has the excellent implementation effect on vibration isolation.

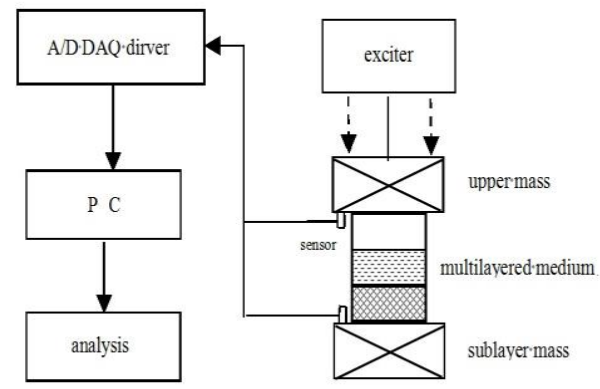

Figure 2. The equipment of test system

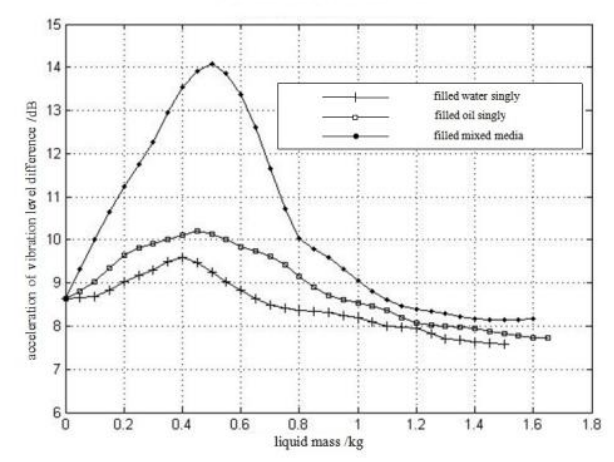

Figure 3. Vibration level difference of acceleration at different media

Using the parameter data shown in Tab 1, absorption coefficient of literature [13] and two-dimensional transfer matrix model in wave-attenuation media can be resolved, then, showing the comparison results in Fig .4. As can be seen from Fig .4, the calculation results of new model are consistent with that of literature [13]. Thus, the rationality of two-dimensional transfer matrix model is verified.

TABle I. PARAmeter TABle

\begin{tabular}{|c|c|c|c|}
\hline Position & $1^{\text {st }}$ Layer & $2^{\text {nd }}$ Layer & $3^{\text {rd }}$ Layer \\
\hline Materials & Dimethicone & Water & $\mathrm{CH}_{2} \mathrm{Cl}_{2}$ \\
\hline $\begin{array}{c}\text { Temperature } \\
{ }^{\circ}\end{array}$ & 20 & 20 & 20 \\
\hline $\begin{array}{c}\text { Sound } \\
\text { Velocity } \\
\times 10^{3} \mathrm{~m} / \mathrm{s}\end{array}$ & 1.040 & 1.483 & 1.070 \\
\hline $\begin{array}{c}\text { Density } \\
\times 10^{3} \mathrm{~kg} / \mathrm{m}^{3}\end{array}$ & 0.963 & 0.998 & 1.330 \\
\hline $\begin{array}{c}\text { Characteristic } \\
\text { Impedance } \\
\times 10^{6} \mathrm{~N} \cdot \mathrm{s} / \mathrm{m}^{3}\end{array}$ & 1.001 & 1.480 & 1.423 \\
\hline $\begin{array}{c}\text { loss factor } \\
\times 10^{-2}\end{array}$ & 2 & 0.1 & 5 \\
\hline
\end{tabular}

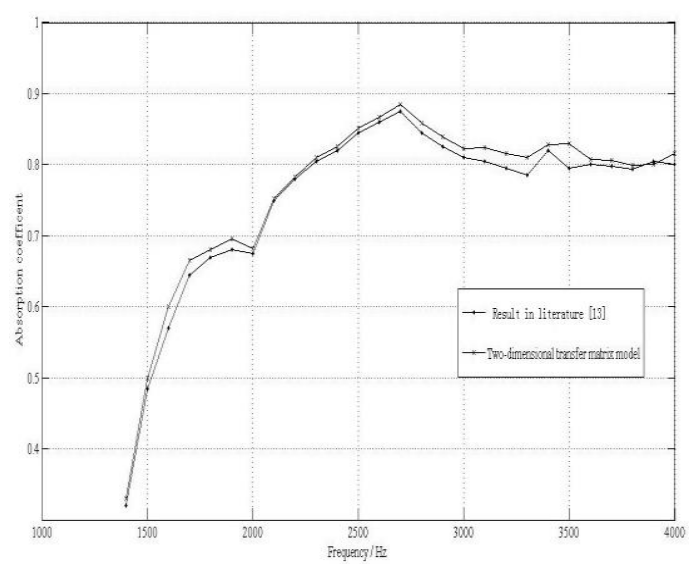

Figure 4. Comparison figure of calculation results

\section{CONCLUSIOnS}

In this paper, the relationship of vibration energy and acoustic field are analyzed, and the conclusion that sound intensity transfer coefficient can be used as the standard for vibration attenuation evaluation of multilayered media is obtained, and then, general 
formulas of transfer coefficient are deduced based on the representation analysis of structure vibration energy and acoustic boundary conditions; The excellent implementation effect on multilayered wave-attenuation vibration isolation is verified by specialized experimental research; Meanwhile, the calculation accuracy of the new model has been verified by comparative analyzing the results of the new model with other literature. In a word, the study not only creates favorable conditions for improving vibration isolation efficiency, but also provides a theoretical and experimental basis for improving vibration isolation theory and developing new isolators.

\section{REFERENCES}

[1] Kong jian-yi, Li gong-fa, Hou Yu, et al. Research on Vibration and Noise Control of Submarine[J]. Noise and vibration control, 2006, 5, pp.1-4.

[2] He lin, Xu wei. Naval Vessel Machinery Mounting Technology and Its Recent Advances[J].Acta Actustica, 2013, 38(2), pp.128-136.

[3] Ibrahim R A. Recent Advances in Nonlinear Passive Vibration Isolators [J].Journal of Sound and Vibration, 2008,314, pp.371-452.

[4] Sun wei-hong, Yan xin. Some Progress of The Research of Vibration and Noise Control Technology for Submarine [J]. Noise and Vibration Control, 2012,5, pp.6-10.

[5] Bu wen-jun, He lin. Alignment Control Strategy for An Air Spring Mounting System of Marine Propulsion
Engine[J].Journal of Vibration and Shock,2012,31(8), pp.112-115.

[6] Zhang shu-zhen, Chen qian, Teng han-dong. Tests for Dynamic Properties of A Gasbag SALIM Vibration Isolator [J].Journal of Vibration and Shock,2012,31(11), pp.98-103.

[7] Courtney W A, Oyadiji S O. Preliminary Investigations into The Mechanical Properties of A Novel Shock Absorbing Electrometric Composite[J].Journal of Materials Processing Technology, 2001, 119(1-3), pp.379- 386.

[8] Teng han-dong, Chen qian. Study on Vibration Isolation Properties of Solid and Liquid Mixture [J].Journal of Sound and Vibration,2009,326(1-2), pp.137-149.

[9] Gao xue, Chen qian. Frequency Response and Jump Avoidance of A Vibration Isolator with Solid and Liquid Mixture[J].Journal of Vibration and Shock, 2012,32(12), pp.150-153.

[10] Ai wei, Jin liang-an. Survey of Warship Mechanical Vibration and Noise Control[J].Journal of Dalian Naval Academy,2014,37(3), pp.77-81.

[11] Ai wei, Jin liang-an, Chi wei. The Status and Prospect of Ship Vibration Isolation Technology [J].Marine Technology, 2015,1, pp.4-8

[12] Shuai chang-geng, He lin, Zhu hai-chao, et al. A Study on the Application and Analysis of a Model with Four-pole Parameters Matrix for Propagation of Acoustic Wave in Multilayered Medium[J]. Noise and Vibration Control,2002,4, pp.7-9.

[13] Yang xue, Wang yuan-sheng, Xu hong-wei. Sound Absorption Properties of Multilayered Polymer Composites for Oblique Incidence[J]. Acta Materiae Compositae Sinica, 2006,23(6), pp.21-28. 